Bull. Austral. Math. Soc.

20D50, 20J05

VoL. 42 (1990) [177-184]

\title{
GEOMETRIC COVERINGS OF GROUPS AND THEIR DIRECTIONS
}

\section{ROLF BRANDL}

\section{To Professor B.H. NeumanN ON his 80Th BIRThdaY}

Let a group $G$ be covered by finitely many disjoint cosets of subgroups $G_{i}$. We study conditions which imply that the subgroups $G_{i}$ are conjugate in $G$.

\section{INTRODUCTION.}

A covering ( $\dagger$ ) of a group $G$ is a representation of $G$ as the union of cosets, so that

$$
G=G_{1} a_{1} \cup \ldots \cup G_{n} a_{n}, \quad \text { where } a_{i} \in G \text { and } G_{1}, \ldots, G_{n} \text { are subgroups of } G \text {. }
$$

For reasons which will become apparent shortly, we call the $G_{i}$ the directions of the covering ( $\dagger$ ).

A celebrated result of B.H. Neumann [8] states that if $(t)$ is irredundant, that is, no coset $G_{i} a_{i}$ can be dispensed with in (†), then all $G_{i}$ are of finite index in $G$. This theorem has applications in various parts of group theory, see for example $[12,11,2$, 3].

Here we consider a special type of coverings that are clearly irredundant (and hence B.H. Neumann's result applies). To avoid trivial cases, we always assume that $n>1$, that is, $G_{i} \neq G$ for all indices $i$.

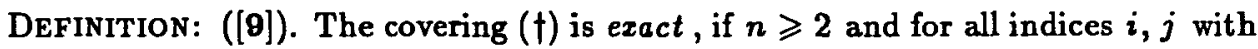
$i \neq j$, we have $G_{i} a_{i} \cap G_{j} a_{j}=\emptyset$.

This means that the covering $(\dagger)$ is exact if and only if every element in $G$ is contained in exactly one of the cosets considered. A very natural example comes from geometry: If $G_{1}$ is a subgroup of finite index $n$ in $G$ (for example, a subspace in a finite vector space), then $G$ is covered by the $n$ right cosets of $G_{1}$ in $G$. In this example, the cosets have all the same direction $G_{1}$, so one could call these parallel.

Our objective here is a study of the directions occurring in an exact covering of some group $G$. First, if $U$ and $V$ are subgroups of finite index in $G$ with $U<V$, then we can cover $G \backslash V$ by disjoint cosets of $V$ and cover $V$ by disjoint cosets of $U$.

Received 19th October 1989

Copyright Clearance Centre, Inc. Serial-fee code: 0004-9729/90 \$A2.00+0.00. 
Thus, we arrive at an exact covering of $G$ along 'very distinct' directions $U$ and $V$. So a natural hypothesis would be to consider only directions that are maximal subgroups (hyperplanes) and ask for connections between the directions.

In general, we cannot expect that they all are equal. To see this, let $S$ be a subgroup of finite index $n$ in $G$ and let $G=a_{1} S \cup \ldots \cup a_{n} S$ be a covering by left cosets. For every $i$, we have $a_{i} S=\left(a_{i} S a_{i}^{-1}\right) a_{i}$ which means that $a_{i} S$ is a right coset of some conjugate of $S$. Hence the question one could ask is, whether the directions in this case are always conjugate. Indeed, for soluble groups this is true as can be seen from part (a) of the following

Theorem A. Let $G$ be a soluble group and let $G=G_{1} a_{1} \cup \ldots \cup G_{n} a_{n}$ be an exact covering of $G$.

(a) If all directions $G_{1}, \ldots, G_{n}$ are maximal subgroups of $G$, then they are conjugate in $G$.

(b) If $G_{1}$ is a maximal subgroup of $G$ and if $\left[G: G_{i}\right]=p^{2}$ for all indices $i$ with $1 \leqslant i \leqslant n$, then all $G_{i}$ are conjugate in $G$.

In general, the directions occurring in an exact covering need not be conjugate, even if they all are maximal subgroups. Indeed, as we shall see below, the symmetric group $S_{5}$ has an exact covering along two directions that are maximal subgroups of index 5 and 10 respectively, so that [9], Theorem 3 cannot substantially be improved upon in the case when $n=5$.

In the above example, the indices of the directions were distinct. As Theorem A shows, this situation cannot occur for soluble groups. Indeed, the latter coverings are geometric in the sense of the following.

DEFinition: The covering $(\dagger)$ is called geometric if it is exact, all directions $G_{i}$ are maximal subgroups and $\left[G: G_{i}\right]=n$ for all indices $i$. We call $n$ the index of the geometric covering $(\dagger)$.

Even for geometric coverings, the directions need not be conjugate in general. As we shall see below, the simple group of order 168 has an exact covering along two nonconjugate directions of index 7 (note that this contrasts with the remark at the end of [9]). However, few conjugacy classes occur if the directions are of prime power index in $G$. It will turn out that the number of conjugacy classes depends on whether or not the prime belongs to a certain set $\omega$ of primes. It seems to be open whether $\omega$ is infinite.

Definition: Let $\omega_{0}$ be the set of all primes $p$ of the form $p=\left(q^{n}-1\right) /(q-1)$ where $q$ is some prime and $n \geqslant 3$, and set $\omega=\omega_{0} \cup\{11\}$. 
The following result depends on the classification of all finite simple groups.

THEOREM B. Let $(\dagger)$ be a geometric covering of prime power index $p^{a}$.

(a) If $a \leqslant 3$ and $p \notin \omega$, then all directions are conjugate.

(b) If $a \leqslant 3$ and $p \in \omega$, then there are at most two classes of directions occurring in ( $\dagger$ ).

(c) For all primes $p$ there exists a group that has a geometric covering of index $p^{a}$ for some $a$, along nonconjugate directions. If $p=2$, we can take $a=4$.

Most of our notation is standard. For a group $G$ and a subgroup $S$, we let Core $G_{G}(S)=\bigcap_{g \in G} S^{g}$. Moreover, $G=[N] Q$ indicates that $G$ is a split extension of a normal subgroup $N$ of $G$ by some complement $Q$. If (†) is a covering of a group $G$, and if $N$ is a normal subgroup of $G$, then we denote by

$$
(\dagger / N) \quad G / N=(G / N) a_{1} N \cup \cdots \cup(G / N) a_{n} N
$$

the induced covering of $G / N$. It is easy to see that if $(\dagger)$ is exact and if $N \leqslant \operatorname{Core}_{G}\left(G_{i}\right)$ for all $i$, then $(t / N)$ is exact.

\section{SOLUBLE GROUPS}

We start with an observation concerning irredundant coverings that easily follows from [8].

Lemma 1. ([8]). Assume that the covering ( $\dagger$ ) is irredundant. Then all $G_{i}$ are of finite index in $G$. If we order the indices of the directions such that $\left[G: G_{1}\right] \leqslant \ldots \leqslant$ $\left[G: G_{n}\right]$, then

(a) $\left[G: G_{1}\right] \leqslant n$.

(b) If $\left[G: G_{1}\right]=n$, then $\left[G: G_{i}\right]=n$ for all indices $i$ and $(\dagger)$ is exact.

The following result is basic for our considerations.

Lemma 2. Assume that the covering $(\dagger)$ is exact. Then

(a) For all indices $i, j$, we have $G_{i} G_{j} \neq G$.

(b) If $G_{1}$ is a maximal subgroup of $G$, then for all indices $i$, we have $\operatorname{Core}_{G}\left(G_{i}\right) \leqslant G_{1}$.

(c) If all directions $G_{1}, \ldots, G_{n}$ are maximal subgroups of $G$, then $\operatorname{Core}_{G}\left(G_{1}\right)=$ $\ldots=$ Core $_{G}\left(G_{n}\right)$. Moreover, the covering $(\dagger) /$ Core $_{G}\left(G_{1}\right)$ is exact.

Proof:

(a) Suppose that $G=G_{1} G_{2}=G_{2} G_{1}$. Then we have $a_{2} a_{1}^{-1}=g_{2} g_{1}$ for some elements $g_{i} \in G_{i}$. We get $G_{2} a_{2}=G_{2} g_{2} g_{1} a_{1}=G_{2} g_{1} a_{1}$ and hence $g_{1} a_{1} \in G_{1} a_{1} \cap G_{2} a_{2}$, a contradiction. 
b) Let $C=\operatorname{Core}_{G}\left(G_{i}\right)$. By (a), we have $G_{1} C \subseteq G_{1} G_{i} \subset G$. As $C$ is normal in $G$, we see that $G_{1} C$ is a subgroup of $G$ and the maximality of $G_{1}$ implies that $C \subseteq G_{1}$ as claimed.

(c) This is clear from (b).

We can now prove our first main result.

Proof of Theorem A: (a) By Lemma 1 and part (c) of Lemma 2, we may assume that $G$ is finite. Part (a) of Lemma 2 implies that $G_{i} G_{j} \neq G$, and finally, a result of $O$. Ore (see [7], p.165) yields that all directions are conjugate.

(b) As above, we may assume that $G$ is finite. By way of contradiction, suppose that $U:=G_{i}$ is not a maximal subgroup for some index $i$. Set $M=G_{1}$.

By part (a) of Lemma 2, we have $M U \neq G$, and part (b) of Lemma 2 implies Core $_{G}(U) \leqslant M$. We proceed by induction to show the weaker claim that $U$ and $M$ are conjugate. Clearly, we may assume that $\operatorname{Core}_{G}(U)=1$. Let $C=\operatorname{Core}_{G}(M)$.

If $C \neq 1$, then $U<C U \leqslant M U<G$. Thus $[G: U]=p^{2}$ implies that $C U$ is a maximal subgroup of $G$. As $[G: M]=p^{2}$, we see that $M$ and $C U$ are nonconjugate and finally, Ore's theorem yields $G=M(C U)=M U$, a contradiction.

Hence $C=1$ and we have $G=[R] M$ for some elementary abelian minimal normal subgroup $R$ of $G$ of order $p^{2}$.

If $p$ does not divide the order of $M$, then both $M$ and $U$ are Hall $\mathrm{p}^{\prime}$-subgroups of $G$ and hence they are conjugate. Thus $M$ is isomorphic to a subgroup of $G L(2, p)$ of order divisible by $p$. An inspection of the subgroups of $P S L(2, p)$ (see [7], p.213 f.) yields that for $p \geqslant 5$, we must have $O_{p}(M) \neq 1$. But this contradicts the faithful and irreducible action of $M$ on $R$. If $p=2$, then $M \cong S_{3}$ and we readily see that $G$ is isomorphic to the symmetric group $S_{4}$, in which case all subgroups of index 4 are conjugate. So let $p=3$. As 3 divides the order of $M$, we see from the structure of $G L(2,3)$ that $M$ contains elements of order 4 , so that a Sylow 2-subgroup of $G$ acts irreducibly on $R$. But $\operatorname{Core}_{G}(U)=1$ and $R \cap U \neq 1$, because otherwise $U$ would be a complement to $R$ and hence it would be conjugate to $M$. Thus we arrive at the contradiction $|U \cap R|=3$, and the result follows.

We now introduce a method that will enable us to construct a series of examples and counterexamples for coverings along two nonconjugate directions. For reasons of simplicity, we have only considered the case of two classes and leave the obvious general case to the reader.

PropositiIon 3. Let $G$ be a group and let $A$ and $B$ be subgroups of finite index in $G$. If $G \neq A B$, then $G$ possesses an exact covering whose directions are conjugate to $A$ and $B$, each of which occur.

Proof: Clearly, the subset $A B$ of $G$ is a disjoint union of certain right cosets of 
$A$ and of certain left cosets of $B$. Hence $G \backslash A B$ is a union of left cosets of $B$ which are right cosets of some conjugates of $B$. The result follows.

The first example shows that part (b) of Theorem $\mathrm{A}$ is no longer true if $\left[G: G_{i}\right]=$ $p^{3}$.

EXAMPLE 4. Let $G=[N] B$ where $B$ is the alternating group of degree 4 and $N$ is an elementary abelian group of order 27 acted upon faithfully and irreducibly by $B$. Then $B$ is a maximal subgroup of $G$ of index 27. Let $C$ be the four-group contained in $B$. Then $N=N_{1} \oplus N_{2} \oplus N_{3}$, where the $N_{i}$ are irreducible modules for $C$. Then $A=\left[N_{1}\right] C$ is a subgroup of index 27 in $G$. Clearly, $A$ and $B$ are nonconjugate in $G$. Moreover, we have $A \cap B=C$, and hence $G \neq A B$, so that Proposition 3 applies.

The second example shows that there are geometric coverings along nonconjugate maximal subgroups. An obvious modification of the argument also shows that the symmetric group $S_{3}$ has an exact covering along maximal subgroups of indices 5 and 10 respectively.

Example 5. Let $p \in \omega$. Then there exists a nonabelian simple group $G$ having two nonconjugate subgroups $A$ and $B$ of index $p$ (see for example [4]). Moreover, $A$ and $B$ are Hall $p^{\prime}$-subgroups of $G$ and hence $p$ does not divide $|A B|$. Thus $A B \neq G$ and Proposition 3 applies.

\section{Prime power indices.}

The proof of Theorem B will be split into a number of lemmas and one proposition. Note that as above, it is sufficient to prove the result under the following.

Hүротнes Is 6. The covering $(\dagger)$ is geometric and Core ${ }_{G}\left(G_{i}\right)=1$ for all $i$.

Thus, $G$ is finite. Let $\left[G: G_{i}\right]=p^{a}$ and let $R$ be a minimal normal subgroup of $G$. Then we have $G=R G_{i}$ for all $i$. If $R$ is abelian, then $G=[R] G_{i}$.

We first consider the case when $R$ is nonabelian. Note that the following result covers the case when $G$ is simple.

LEMma 7. Assume that Hypothesis 6 holds and let $R$ be a product of $t$ nonabelian simple groups.

(a) If $p \notin \omega$, then all directions $G_{i}$ are conjugate in $G$.

(b) If $p \in \omega$ and $a \leqslant 3$, then the $G_{i}$ fall into $\leqslant 2$ conjugacy classes in $G$.

Proof: Let $R=R_{1} \times \cdots \times R_{t}$ where the $R_{i}$ are nonabelian and simple and set $D_{i}=R \cap G_{i}(1 \leqslant i \leqslant t)$. Then clearly, $D_{i}$ is normal in $G_{i}$ and so $\operatorname{Core}_{G}\left(G_{i}\right)=1$ implies that

$$
G_{i}=N_{G}\left(D_{i}\right)
$$


Thus, it suffices to show that the groups $D_{1}, \ldots, D_{n}$ fall into at most two conjugacy classes.

For this, we consider the intersections $S_{i, j}=D_{i} \cap R_{j}$ of $D_{i}$ with the direct factors of $R$. First, observe that $S_{i, j} \neq R_{j}$, because otherwise $R_{j} \leqslant G_{i}$, and so $S_{i, j}^{G}=S_{i, j}^{R G_{i}} \leqslant R_{j}^{G_{i}} \leqslant G_{i}$ which contradicts $\operatorname{Core}_{G}\left(G_{i}\right)=1$. Moreover, ([5], p.314) implies that

$$
D_{i}=S_{i, 1} \times \cdots \times S_{i, t}
$$

and hence $\left[R_{j}: S_{i, j}\right]$ is a proper power of $p$ for all $i, j$.

If $p \notin \omega$, then by [4] (see also [5], p.314), all $S_{i, j}$ are conjugate in $R_{j}$. Therefore, all $D_{i}$ are conjugate in $R$ and part (a) follows from (1).

So let $p \in \omega$ and assume $a \leqslant 3$. Then the above shows that $R$ is a direct sum of $t \leqslant 3$ simple groups. The case $t=1$ clearly follows from [4], so let $2 \leqslant t \leqslant 3$. Now $R$ is a minimal normal subgroup of $G$, and hence $G=R G_{i}$ acts transitively on the set $\left\{R_{1}, \ldots, R_{t}\right\}$. As $t$ is a prime, this implies that there exists $x \in G_{i}$ such that

$$
R_{j}{ }^{x}=R_{j+1}(\text { read indices mod } \mathrm{t}) \text {. }
$$

Assume that the direct summands $S_{i, j}$ and $S_{k, j}$ of $D_{i}$, respectively $D_{k}$ are conjugate in $R$ (or, equivalently, are conjugate in $R_{j}$ ). We proceed to show that $D_{i}$ and $D_{j}$ are conjugate in $R$. we have

Indeed, let $S_{k, j}=S_{i, j}^{r}$ for some $r \in R$ and some index $j$. Then by (2) and (3),

$$
S_{k, j+1}=S_{k, j}^{x}=S_{i, j}^{r x}=S_{i, j}^{x \&}=S_{i, j+1}^{*}
$$

with $s=r^{2} \in R$. Hence $S_{k, j+1}$ and $S_{i, j+1}$ are conjugate in $R_{j+1}$. This is true for every index $j$, and hence $D_{i}$ and $D_{k}$ are conjugate in $R$. The result now follows from [4] and (1).

The case when $R$ is abelian is dealt with in the next two lemmas.

Lemma 8. Assume that Hypothesis 6 holds and let $R$ be abelian. If $G_{1}$ has a nontrivial abelian normal subgroup, then all $G_{i}$ are conjugate.

Proof: Let $Q=G_{i}$ and let $A$ be a minimal abelian normal subgroup of $Q$. As $Q$ acts faithfully and irreducibly on $R$, we see that $A$ is a $q$-group for some prime $q \neq p$. Let $S=R A$. Then $A$ is a Sylow $q$-subgroup of $S$. Moreover, $Q \leqslant N_{G}(A)$ and Dedekind's law yields $N_{G}(A)=\left[N_{R}(A)\right] Q$. Now $N_{R}(A)=C_{R}(A)$ clearly is $Q$. invariant, and hence we have $N_{R}(A)=1$, that is, $Q=N_{G}(A)$. As all Sylow $q$ subgroups of $S$ are conjugate in $S$, we see that all $G_{i}$ are conjugate in $G$. 
Lemma 9. Assume that Hypothesis 6 holds and assume that $R$ is abelian. If $a \leqslant 3$, then all $G_{i}$ are conjugate.

Proof: By Lemma 8, we only have to consider the case when $Q:=G_{i}$ does not have any nontrivial abelian normal subgroups. By Theorem $A$, we may assume that $Q$ is nonsoluble. If $a \leqslant 2$, then $Q$ is isomorphic to a subgroup of $G L(2, p)$. If $p$ divides $|Q|$, then either $p \leqslant 3$ in which case $G$ were soluble. Otherwise, $p \geqslant 5$ and $Q \geqslant S L(2, p)$. But in this case, $Z(Q) \neq 1$ and Lemma 8 applies.

So let $a=3$. First consider the case when $p$ is odd. If $p$ does not divide $|Q|$, then the result follows from the theorem of Schur-Zassenhaus, so assume that $p$ divides $|Q|$. An inspection of the list given in [1] and using the fact that $Q$ acts irreducibly on $R$, shows that we must have $S L(3, p) \leqslant Q \leqslant G L(3, p)$. An inspection of the subgroups of $G L(3,2)$ shows that the same is true for $p=2$.

If $p \equiv 1(3)$, then $Q$ has a nontrivial abelian normal subgroup and we are done by Lemma 8. So assume $p \not \equiv 1(3)$. Let $T_{0}$ be a complement to $R$ in $G$ and assume that $Q$ and $T_{0}$ are nonconjugate in $G$. We show that $G=Q T_{0}$. The result then follows from part (a) of Lemma 2. Clearly, it is sufficient to prove the latter statement for the subgroups of $Q$, respectively $T_{0}$, isomorphic to $S L(3, p)$, so let $Q=S L(3, p)$. By way of contradiction, assume that $G \neq Q T_{0}$. Then for every conjugate $T$ of $T_{0}$, we have $G \neq Q T$ (see [7], p.675). Now $G$ contains a cyclic Hall subgroup $H$ of order $p^{2}+p+1$ of $Q$ (a so-called Singer-cycle). As nilpotent Hall-subgroups are conjugate, we may assume that $Q \cap T \geqslant H$. An inspection of all subgroups of $Q$ containing $H$ and using [6] yields $|Q \cap T| \leqslant 3\left(p^{2}+p+1\right)$. Thus, we arrive at

$$
|Q T|=\frac{|Q| \cdot|T|}{|Q \cap T|} \geqslant p^{s} \cdot|Q|=|G|,
$$

a contradiction.

Parts (a) and (b) of Theorem B now follow from Lemmas 7-9. For part (c), we finally prove the following.

Proposition 10. For every prime $p$, there exists a group $G$ having a geometric covering of index $p^{a}$ for some a depending on $p$ along nonconjugate directions.

Proof: If $p \leqslant 3$, let $Q=A_{5}$, otherwise, let $Q=A_{p}$. By a result of Stammbach [10], there exists an irreducible $G F(p) Q$-module $M$ such that $B^{1}\left(Q / C_{Q}(M), M\right) \neq 0$. As $Q$ is simple, $M$ must be faithful and so $H^{1}(Q, M) \neq 0$. Let $G=[M] Q$ be the natural split extension and let $R$ be a complement to $M$ that is not conjugate to $Q$. We show $Q R \neq G$. Otherwise, $G=Q R$ and hence $[Q: Q \cap R]=|M|=p^{a}$. As $Q=A_{p}$, this would imply $|M|=p$, a contradiction. Note that for $p=2$, the faithful and irreducible modules for $A_{5}$ are of dimension 4 , so $a=4$ and the result follows. 


\section{REFERENCES}

[1] D.M. Bloom, 'The subgroups of PSL(3,q) for odd q', Trans. Amer. Math. Soc. 127 (1967), 150-178.

[2] R.D. Blyth, 'Rewriting products of group elements - I', J. Algebra 116 (1988), 506-521.

[3] M. Curzio, P. Longobardi, M. Maj and D.J.S. Robinson, 'A permutational property of groups', Arch. Math. 44 (1985), 385-389.

[4] R. Guralnick, 'Subgroups of prime power index in a simple group', J. Algebra 81 (1983), 304-311.

[5] R. Guralnick, 'Subgroups inducing the same permutation representation', J. Algebra 81 (1983), 312-319.

[6] M.D. Hestenes, 'Singer groups', Canad. J. Math. 22 (1970), 492-513.

[7] B. Huppert, Endliche Gruppen I (Springer Verlag, Berlin-Heidelberg-New York, 1967).

[8] B.H. Neumann, 'Groups covered by permutable subsets', J. London Math. Soc. 29 (1954), 236-248.

[9] M.M. Parmenter, 'Exact covering systems for groups', Fund. Math. 123 (1984), 133-136.

[10] U. Stammbach, 'Cohomological characterisations of finite solvable and nilpotent groups', J. Pure Appl. Algebra 11 (1977/78), 293-301.

[11] M.J. Tomkinson, 'FC-groups', in Research Notes in Mathematics (Pitman, London, 1984).

[12] G. Zacher, 'A lattice characterization of the index of a subgroup in a group', Atti Accad. Naz. Lincei Rend. Cl. Sci. Fis. Mat. Natur. (8) 69 (1980), 317-323.

Mathernatisches Institut

Am Hubland 12

D-8700 Würzburg

West Germany 